\title{
DISEÑO INSTRUCCIONAL Y EVALUACIÓN ADAPTATIVA EN TIEMPOS DE COVID-19
}

\author{
Yanira Xiomara de la Cruz Castañeda ${ }^{1}$ \\ Universidad Autónoma de Zacatecas/México \\ https://orcid.org/0000-0002-8696-6724
}

\begin{abstract}
RESUMEN:
El objetivo de este artículo es analizar, mediante el desempeño y aprendizaje de un grupo de estudiantes de posgrado del Doctorado en Gestión Educativa y Políticas Públicas de la Universidad Autónoma de Zacatecas, México, la implementación de estrategias pedagógicas adaptativas incorporadas en la asignatura Liderazgo y Gestión Educativa como parte de los contextos de la Educación a Distancia iniciados tras la suspensión de las clases presenciales, como medida sanitaria para prevenir los contagios por COVID-19, para determinar las características del proceso enseñanza-aprendizaje-evaluación que pueden ser replicadas en escenarios similares de instituciones de nivel superior. Se propone un abordaje cualitativo, siendo el eje de análisis el uso, gestión e incorporación de nuevas estrategias didácticas que promueven el trabajo autónomo, la creatividad y el pensamiento crítico en vez del tradicional instruccionismo que impera en este nivel educativo. Para la recolección de información y el análisis pertinente, se compilaron los datos de la plataforma Moodle que contiene la propuesta curricular adaptada del seminario, se incluyeron variables, factores e indicadores sobre los estudiantes. Los resultados mostraron buen desempeño académico, cumplimiento y participación asertiva, lo que permitió el logro de los objetivos planteados; sin embargo, existen áreas de oportunidad en actitudes y valores de los estudiantes. La investigación permitió ampliar el conocimiento sobre los resultados de la implementación de estrategias adaptativas que utilizan tecnologías y recursos web como elementos de mediación y evaluación formativa, beneficiando la planificación y organización de actividades que logren los objetivos curriculares planteados desde una participación activa de los alumnos, atendiendo a la diversidad y promoviendo contextos educativos democráticos.
\end{abstract}

Palabras clave: Estrategias Adaptativas. Diseño Instruccional. TIC. Educación a Distancia. COVID.

\section{INSTRUCTIONAL DESIGN AND ADAPTIVE ASSESSMENT IN TIMES OF COVID-19}

\begin{abstract}
:
The objective of this article is to analyze, through the performance and learning of a group of graduate students of the Doctorate in Educational Management and Public Policies of the Universidad Autónoma de Zacatecas, Mexico, the implementation of adaptive teaching strategies incorporated in the subject Leadership and Educational Management as part of the Distance Education contexts initiated after the suspension of face-to-face classes, as a health measure to prevent COVID-19 infections, to determine the characteristics of the teaching-learning-evaluation process that can be replicated in similar scenarios in higher level institutions. A qualitative approach is proposed, being the axis of analysis the use, management and incorporation of new didactic strategies that promote autonomous work, creativity and critical thinking instead of the traditional instructionism that prevails at this educational level. For the collection of information and the pertinent analysis, data were compiled from the Moodle platform containing the curricular proposal adapted from the seminar, variables, factors and indicators on students were included. The results showed good academic performance, compliance and assertive participation,
\end{abstract}

1 Doctora en Investigación Educativa. Línea de investigación en Tecnología Educativa. Adscrita a la Unidad Académica de Docencia Superior de la Universidad Autónoma de Zacatecas. Actualmente docente investigadora en el Doctorado en Gestión Educativa y Políticas Públicas. Perfil PRODEP. México. E-mail: xiomara@uaz.edu.mx 
which allowed the achievement of the proposed objectives; however, there are areas of opportunity in attitudes and values of the students. The research allowed expanding the knowledge on the results of the implementation of adaptive strategies that use technologies and web resources as elements of mediation and formative evaluation, benefiting the planning and organization of activities that achieve the curricular objectives from an active participation of the students, attending to diversity and promoting democratic educational contexts.

Keywords: Adaptive strategies. Instructional design. ICT. Distance education. COVID.

\section{DESIGN INSTRUCTIONAL E AVALIAÇÃO ADAPTATIVA EM TEMPOS DE COVID-19}

\section{RESUMO:}

Este artigo objetiva analisar, através da performance e aprendizado de um grupo de estudantes do Doutorado em Gestão Educacional e Políticas Públicas da Universidad Autónoma de Zacatecas, México, a implementação de estratégias de ensino adaptativas incorporadas na disciplina Liderança e Gestão Educacional como parte do contexto da Educação a Distância após a suspensão das aulas presenciais, como medida de prevenção das infecções por COVID-19, para determinar as características do processo ensino-aprendizado-avaliação o qual pode ser replicado em cenários similares nas instituições de ensino superior. É proposta uma abordagem qualitativa, sendo o eixo de análise, o uso, gestão e incorporação de novas estratégias didáticas que promovem o trabalho autônomo, criatividade e pensamento crítico ao invés do instrucionismo que predomina neste nível educacional. Para a coleta de informações e análise pertinente, os dados foram compilados da plataforma Moodle contendo a proposta curricular adaptada do seminário, foram incluídos variáveis, fatores e indicadores sobre os alunos. Os resultados demonstram boa performance acadêmica, presença e participação assertiva, o que permitiu a obtenção dos objetivos propostos; entretanto, à áreas de oportunidade nas ações e valores dos estudantes. A pesquisa permitiu ampliar o conhecimento sobre os resultados da implementação das estratégias adaptativas que utilizam tecnologias e recursos web como elemento de mediação e avaliação formativa, beneficiando o planejamento e organização das atividades que cumprem os objetivos curriculares através da participação ativa dos estudantes, atendendo às diversidades e promovendo contextos educacionais democráticos.

Palavras-chave: Estratégias Adaptativas Design Instrucional. TIC. Educação a distância. COVID.

\section{Introducción}

La suspensión de actividades presenciales en el ámbito educativo internacional de todos los niveles como medida de emergencia ante la pandemia de enfermedad por coronavirus (COVID-19) ha sido uno de los retos más importantes por mantener la continuidad del aprendizaje en los estudiantes. En el proceso de adaptación, flexibilización y contextualización curricular, los docentes, al ser los mediadores entre familias y autoridades educativas, se han enfrentado a diversas problemáticas para adaptar sus prácticas a la modalidad a distancia, utilizando en mayor medida sus habilidades (conocidas o aprendidas) para las Tecnologías de Información y Comunicación (TIC), la implementación de estas estrategias y cambios en los diseños curriculares de todos los niveles en la educación mexicana está apenas iniciando, su uso es muy desigual y los profesores al implementarlas han expresado su inconformidad debido a la falta de infraestructura 
necesaria y la poca capacitación acorde a este escenario para el sector. Estudios previos describen las situaciones que los docentes identificaron al enfrentarse a la tarea de "mudar" su espacio de acción a la virtualidad. Específicamente, en nivel superior en México, de acuerdo a una encuesta aplicada a los profesores de la Universidad Autónoma de México (UNAM) en marzo de 2020 donde se les solicitó propuestas concretas para que la universidad a través de sus autoridades los apoyarán con su labor ante la contingencia, las respuestas versaron en las siguientes categorías a reforzar: disponibilidad de recursos digitales, formación pedagógica, recursos informativos sobre uso de tecnologías en educación, mejora de procesos institucionales, acompañamiento pedagógico, asesoría técnica e información de los recursos institucionales (CEPAL, 2020).

Ante este escenario, la implementación de estrategias que promuevan el aprendizaje desde un enfoque diferente a la migración de la metodología tradicional en espacios virtuales (práctica común en estos momentos), como la descrita en este artículo, es importante difundirla y analizarla para comprender las características que marcarán el inicio de nuevas formas de interacción como fenómeno social en el distanciamiento educativo y su paulatino regreso a clases presenciales, que, definitivamente, no serán iguales.

A partir de este esquema, se propone el objetivo de este artículo centrado en analizar mediante el desempeño y aprendizaje de un grupo de estudiantes de posgrado del Doctorado en Gestión Educativa y Políticas Públicas de la Universidad Autónoma de Zacatecas, México, la implementación de estrategias adaptativas incorporadas en la asignatura Liderazgo y Gestión Educativa como parte de los contextos de la Educación a Distancia (EaD) implementados tras la suspensión de las clases presenciales, para determinar las características del proceso enseñanza-aprendizaje-evaluación que pueden ser replicadas en escenarios similares de instituciones de nivel superior del país y a nivel mundial; propiciando la reflexión sobre la importancia de estos espacios para analizar las características de la nueva forma de presentar los contenidos, procesos, actitudes y valores, la preparación de los estudiantes para comprender la realidad, convivir y actuar en tiempos de crisis e incertidumbre, a nivel individual y familiar e impulsar soluciones colectivas a desafíos urgentes que contribuyan a la transformación estructural del sistema educativo actual.

\section{Prácticas pedagógicas vs Diseño instruccional}

Con la incorporación de las TIC como elemento mediador principal para la promoción del aprendizaje en los espacios de formación académica virtual como parte del replanteamiento del ejercicio docente a consecuencia de la crisis sanitaria a nivel mundial por COVID-19, el sistema 
educativo encontró más que nunca áreas de oportunidad y se enfrentó a retos que sobreponen las voluntades y capacidades del sector, indudablemente, la falta de infraestructura para el acceso a las tecnologías e internet es muestra de ello; sin embargo, a la par de estas desavenencias, existen profesores preocupados por su comunidad y, hoy más que nunca, la creatividad y vocación por la profesión ha comenzado a dar frutos. Indudablemente, el cambio no sucede con el actuar de manera aislada, sin embargo, este tipo de prácticas pedagógicas disruptivas que "salen del molde" del discurso pesimista sobre lo que falta y se concentran en lo que se puede hacer, son un tema que merece ser estudiado.

Comencemos por comprender que las prácticas pedagógicas se definen como lo opuesto a la teoría educativa, que se compone del saber sistemático y formal sobre los procesos de aprendizaje, enseñanza y evaluación a partir de la investigación y la contrastación entre pares. La teoría es el conocimiento base de la enseñanza (Shulman, 1987). Las prácticas pedagógicas, en cambio, es lo que hacen cotidianamente los docentes con los alumnos, principalmente en las aulas (Álvarez, 2015). Son las actividades que se hacen en las escuelas y que pueden o no estar basadas en un cuerpo teórico concreto. Por ejemplo, hay docentes que enseñan siguiendo a los maestros que tuvieron en la primaria, secundaria, educación media o universidad, y no a partir de una teoría fundamentada o la investigación. Lo que genera un atraso y promueve el instruccionismo que tanto daño está haciendo a nuestros estudiantes, principalmente por la desarticulación entre lo que se aprende en la escuela y las exigencias de la sociedad.

Desde la Socioformación (Ambrosio, 2018; Tobón, 2017b), las prácticas pedagógicas se definen como acciones colaborativas que se implementan entre diferentes actores (docentes, directivos, asesores y comunidad) para que los estudiantes (y demás integrantes de las instituciones educativas) aprendan a resolver problemas del contexto mediante la gestión y cocreación del conocimiento a partir de fuentes pertinentes, la articulación de diferentes saberes y el mejoramiento continuo en un entorno de inclusión, de tal manera que se contribuya a transformar las condiciones de vida y se aporte a la sostenibilidad ambiental. En este sentido, en la socioformación las prácticas pedagógicas no son exclusivas de los docentes sino de todos los actores vinculados a los centros de formación.

Como se puede observar, las prácticas docentes están relacionadas a la conceptualización anterior al ser entendidas como las intervenciones que realiza el profesor para el aseguramiento del aprendizaje y el logro de los objetivos, además, son expresadas a través de actividades que permiten la construcción del conocimiento, en la configuración de nuevos entornos de enseñanza-aprendizaje y en la transformación de la cultura escolar y docente (Tobón, 2018). No se trata del 
cumplimiento de un rol como tal, sino que promueve la innovación y es en ellas donde suceden los cambios estructurales que se ponen en práctica dentro de los espacios áulicos.

Ahora bien, cuando se delinea un curso, los materiales y la metodología a utilizar, es importante explicitar un concepto que ha sido objeto de controversia desde su creación, debido principalmente al alcance y personal implicado en él: Diseño Instruccional. Con el auge del uso de la tecnología en la educación, es en este proceso donde se desglosan los factores determinantes para la promoción de la calidad y el logro de los objetivos de los planes y programas de estudio.

Este concepto fue introducido por Robert Glaser en 1960, desde la perspectiva de diversos autores podría definirse como: La planeación, la preparación y el diseño de los recursos y ambientes necesarios para que se lleve a cabo el aprendizaje (Bruner,1960). La disciplina interesada en prescribir métodos óptimos de instrucción, al crear cambios deseados en los conocimientos y habilidades del estudiante (Reigeluth,1983). Planificación instruccional sistemática que incluye la valoración de necesidades, el desarrollo, la evaluación, la implementación y el mantenimiento de materiales y programas (Richey, Fields y Foson, 2001). Entre muchas otras.

Existen varios modelos de diseño instruccional que los agrupan según los orígenes y los propósitos con que fueron formulados. Actualmente, una de las corrientes más fuertes lo fundamenta en la tecnología educativa y se entiende como el diseño y uso de las TIC para la elaboración de recursos de aprendizaje, así como a la planificación de la educación que implica elaborar guiones, planes y programas, y que generalmente se lleva a cabo bajo proyectos estandarizados (Serrano \& Ponds, 2008), lo que se traduce en un campus de acción didáctica que conlleve a la articulación entre el estudiante, el docente y los contenidos para gestionar el proceso de aprendizaje.

De esta manera la $\mathrm{EaD}$, forma principal de enseñanza actual debido a la contingencia sanitaria, integra entre sus componentes: alumno, docente, comunicación, estructura, modelo educativo y planes de estudio (García, 1999). El alumno, como destinatario, debe tener un proceso educativo eficaz, por lo que es necesario la creación de sistemas (planteamientos metodológicos) que se transformen-cambien de forma significativa, ya que la mayor parte del proceso lo transitan de manera autónoma e independiente. El conocimiento e implementación adecuada por parte de los docentes de las prácticas educativas a través de diseños instruccionales acordes a las características de nuestros días, es ya una necesidad.

Finalmente, es cierto que el aseguramiento de la calidad a partir del aislamiento preventivo en todos los países involucra un cambio que compete principalmente ámbitos de política pública, demanda que el sistema educativo como tal otorgue los lineamientos y condiciones 
de operación acordes al contexto de nuestras escuelas, la participación de más actores como supervisores, directores, padres de familia y sociedad en general; sin embargo, el escenario de concreción e impacto significativo sucede "con el operar directo del docente", siendo éste quien advierte de primera impresión los problemas y sus tipos. Es entonces el sector de profesores, los que tenemos el reto en puerta, por lo que se necesitan realizar investigaciones que involucren propuestas de acción docente de todos los niveles y contextos educativos, fortalecer redes de colaboración interinstitucionales, haciendo uso de elementos al alcance para un diseño curricular efectivo, acorde a tiempos y recursos humanos y tecnológicos disponibles.

\section{Enseñanza y evaluación adaptativa}

El sistema educativo de México, como el de varios países de América Latina, cuenta con amplia diversidad sobre el origen étnico, de género, religión, orientación sexual, discapacidad, etc., de los estudiantes y es injerencia del Estado proporcionar educación de carácter obligatoria a todos los habitantes del país, sin importar estas características, incrementando su cobertura y favoreciendo la calidad del aprendizaje; siendo el derecho de acceso a la educación uno de los mayores retos para el ámbito educativo, ya que éste goza de una historia que pone de manifiesto la marginación y segregación de grandes colectivos humanos en situaciones de desigualdad educativa (mujeres, personas pertenecientes a minorías culturales o etnias, clases sociales desfavorecidas y con diversidad de capacidades, etc.). ¿Qué sucede cuando las condiciones en contextos rurales son abismalmente diferentes a las de entornos urbanos?, más aún, cuando el acceso a una buena señal de internet, a la luz eléctrica, es casi imposible. Nuevamente, es el sector docente el encargado de promover un aprendizaje integral, es responsable de la creación e integración de recursos y estrategias que satisfagan las necesidades educativas especiales y promuevan la inclusión, utilizando como apoyo las TIC, respetando la riqueza de nuestra sociedad pluricultural y multilingüística, así como los saberes que se han construido y preservado a lo largo de la historia de nuestro país.

Estudios pre y post pandemia han puesto de manifiesto que el profesorado, si bien se siente preparado en conocimiento y dominio de los contenidos, no está preparado para atender la diversidad de situaciones ante la realidad actual, considerando que los recursos gestionados por las administraciones son insuficientes. Sin embargo, conscientes de la necesidad de estrategias innovadoras eficaces, están llevando a cabo estrategias pedagógicas a partir de principios de Educación Adaptativa (García, 2000), tomando acción directa como se mencionó en el apartado anterior. 
El término Educación adaptativa es acuñado por primera vez en el año 1977 con la aparición del libro Adaptive Education de Glaser, pone de manifiesto que los problemas de aprovechamiento de los estudiantes se deben a la falta de alineación entre los procedimientos educativos utilizados y las características de los estudiantes, principalmente las que nacen en los propios contextos. Como algunos autores dicen (Parrilla, 2002; Booth, 2000) se trata de una "reforma inclusiva" que plantea qué medidas puede articular el sistema educativo para responder a la igualdad desde la diversidad, considerando que la diversidad en la escuela supone la participación de cualquier persona, con independencia de sus características sociales, culturales, biológicas, intelectuales, afectivas, etc., luchando contra las barreras del aprendizaje en la escuela y a favor de una educación de calidad para todos los alumnos, lo que supone, en definitiva, una educación que acepte las diferencias y sepa utilizarlas como elemento de enriquecimiento mutuo en lugar de servir como elemento marginador o segregador (Zabalza, 1999).

La educación adaptativa considera que hay siete elementos interrelacionados que hacen que una estrategia de adaptación funcione (Martínez, 2008) o, por el contrario, no tenga éxito:

1) un marco teórico potente que sustente y de forma a la manera de abordar el problema y la solución; 2) un planteamiento de la diversidad desde una cultura positiva que considere las diferencias como una realidad a la que hay que atender. Afirma que la escuela tiene capacidad de dar respuesta a la diversidad para que no sea un impedimento en el logro de los objetivos planteados. La comunidad educativa debe crear un entorno de seguridad, aceptación y colaboración entre todos; 3) la formación específica del profesorado en atención a la diversidad que les implique competentemente en el diseño de soluciones y recursos; 4) un trabajo cooperativo e interprofesional que incluya técnicos en intervención psicopedagógica y docentes de distintos ámbitos con experiencia educativa; 5) un diagnóstico de la realidad escolar y de las diferencias de los estudiantes y del sistema, identificando todos aquellos elementos que facilitan o, por el contrario, impiden el logro de los resultados esperados; 6) la búsqueda e introducción de estrategias y recursos variados y eficaces; y 7) el apoyo y flexibilidad de la dirección del centro y de la administración educativa que facilite la reorganización del entorno escolar según las necesidades de la situación particular (p.256).

Exceptuando el último punto, nuevamente los elementos se vinculan con el actuar de los docentes (enseñanza).

De acuerdo con la cartografía conceptual previamente descrita, estamos en condiciones de hablar sobre Enseñanza adaptativa, ésta se considera como una práctica fundamental que ejecutan los docentes sobresalientes o de impacto (Tobón, 2018). Consiste en ajustar los procesos de mediación de acuerdo con las necesidades de aprendizaje de los alumnos. Por ejemplo, 
Parsons \& Vaughn (2016) plantean que los docentes que tienen impacto adaptan la enseñanza a los alumnos. A partir de la reflexión, los docentes identifican las necesidades de los estudiantes antes, durante y al final de la mediación, y con base en ello implementan ajustes en las actividades de aprendizaje, enseñanza y evaluación (Parsons et al., 2018). En la socioformación, más que enseñanza adaptativa se aborda el concepto de formación adaptativa, e implica, por una parte, los ajustes que hace continuamente el docente en el proceso de mediación (y la forma cómo se van dando las actividades de aprendizaje, evaluación y gestión de los recursos), como también el mismo estudiante y sus pares, para tener impacto en la resolución de los problemas del contexto mediante la autoevaluación de las acciones y el mejoramiento continuo. Requiere identificar las necesidades y potenciales de los alumnos como también los recursos que se pueden gestionar.

Como se ha mencionado, la enseñanza adaptativa pretende que los estudiantes alcancen los objetivos propuestos en planes y programas educativos a través del diseño de recursos acordes a sus características, sin embargo, el proceso que se necesita abordar y que interfiere directamente en los resultados del aprendizaje, es la evaluación.

El concepto evaluación, al tener sus inicios en el ámbito empresarial, suele tener connotación cuantitativa, medible, objetiva y demostrable. En el ámbito educativo, la evaluación como proceso consistente en la comprobación del mero logro de unos objetivos o conocimientos (Tyler, 1950) debió haber evolucionado hacia una concepción de asesoramiento, regulación, reorientación y ordenación de los aprendizajes, para mejorar el proceso de enseñanza-aprendizaje (Castillo \& Cabrerizo, 2009). Eliminar el enfoque finalista, punitivo e incorregible adquiere especial importancia debido a las interacciones y escenarios educativos actuales, constituye un proceso que requiere ser dosificado a lo largo del diseño instruccional planteado y no solamente en momentos específicos, por lo regular finales. Es un concepto más amplio que el de sólo medir o calificar, incide en tres momentos específicos (García, 2020): 1) tareas auténticas a realizar por los estudiantes (útiles, orientadas a la profesión), 2) realimentación al estudiante y 3) participación de todos los agentes en la evaluación a través de autoevaluación, heteroevaluación, evaluación por pares y coevaluación (mezcla de las anteriores).

Por lo anterior, definir el enfoque de evaluación para una asignatura o curso es un aspecto integrador del proceso de enseñanza-aprendizaje, debe ser analizado y diseñado con la misma atención y tomando en cuenta las condiciones propias del contexto, adquiriendo el papel adaptativo que mencionamos en párrafos anteriores. En la siguiente imagen se presenta un esquema (Figura 1) que clasifica las principales acepciones de la evaluación orientada al aprendizaje: 
Figura 1. Dimensiones de la evaluación orientada al aprendizaje.

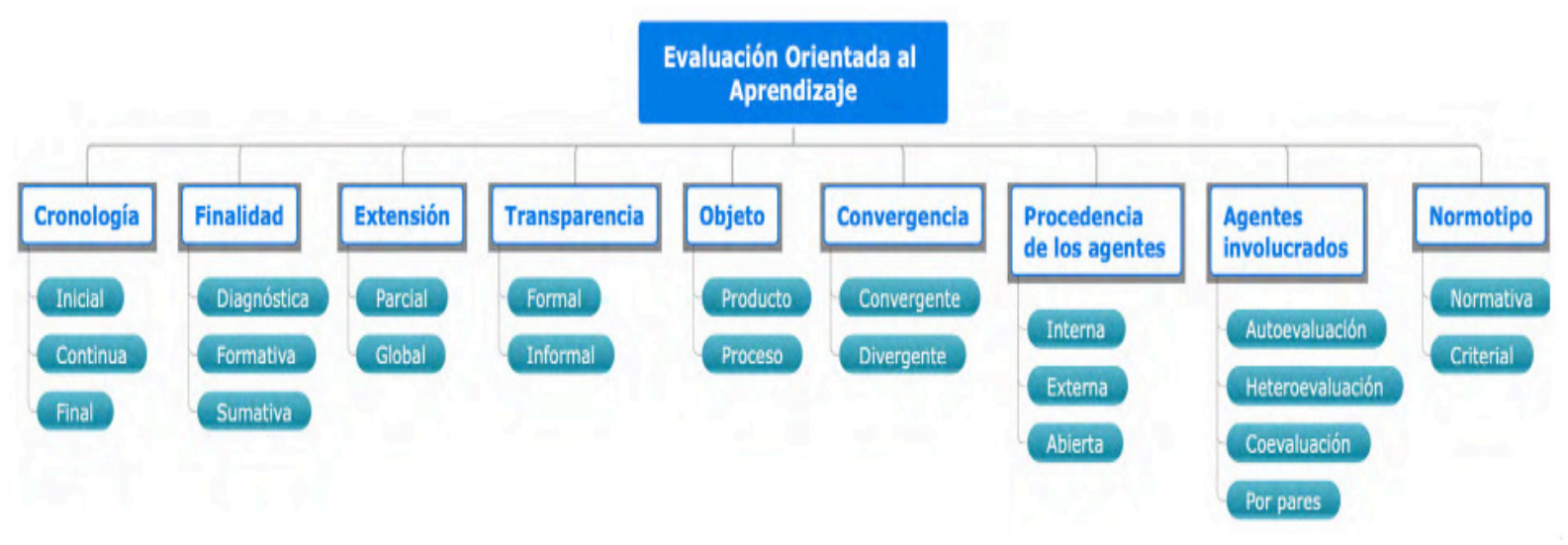

Fuente: García, 2020.

Como se puede observar, el uso de este proceso es más complejo y requiere un nivel de análisis mayor al que comúnmente realiza el docente, éste debe tomar en cuenta elementos como cronología, actores involucrados, finalidad o intención, convergencia, normotipo, etc.

La finalidad o intención de la evaluación, establecerá el tipo y momento para utilizarla, debe tomarse en cuenta además los objetivos y propósitos de la asignatura, así como las estrategias de enseñanza-aprendizaje a implementar, proceso que se vuelve cíclico (Figura 2) entre estos elementos y que deben estar alineados para determinar la secuencia del curso:

Figura 2. Triangulación entre propósitos, estrategias de enseñanza-aprendizaje y evaluación.

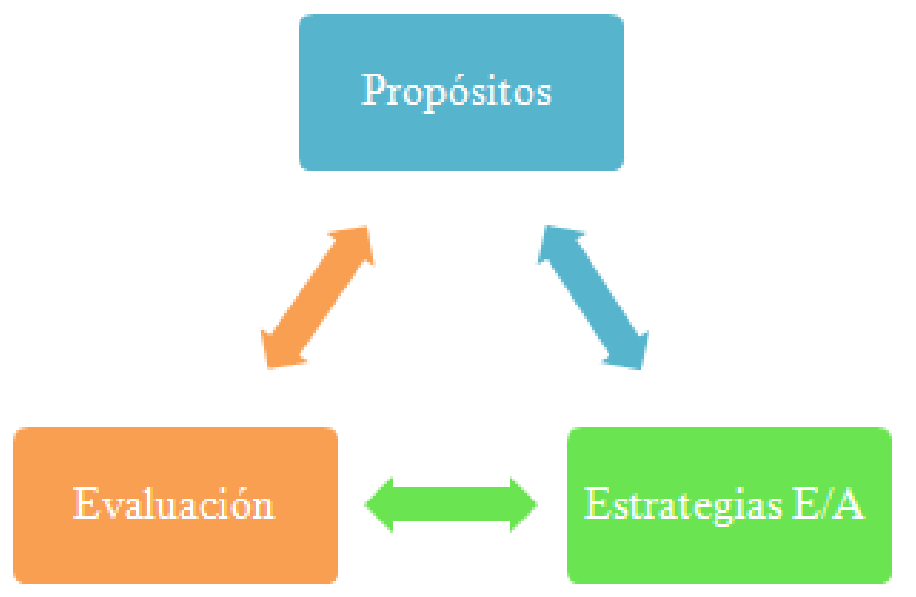

Fuente: Elaboración propia. 
Otro aspecto importante es el diseño de instrumentos de evaluación del desempeño del estudiante que vayan más allá de los exámenes pregunta/respuesta, situaciones que por lo regular limitan al estudiante al priorizar el aprendizaje memorístico, ya que éste responde conforme a lo que el docente solicita "nada más"; implementando instrumentos que desafíen lo visto en las sesiones y materiales, que le permitan crear, argumentar, cuestionar su realidad, etc., y que estén adaptados a los propósitos, es que avanzaremos hacia nuevas formas de aprender y hacer uso del conocimiento, logrando la convergencia de las teorías pedagógicas orientadas por las características del contexto, modalidad, nivel educativo y la incorporación de la tecnología como agente dinamizador del proceso educativo.

Así pues, la evaluación adaptativa (caracterizada en este texto) proporciona estructura al aprendizaje, ya que permite descomponer el todo en partes manejables, facilitando a los alumnos una visión de sus progresos, incluyendo el desarrollo de su comprensión y dominio de competencias; es alentadora, motivante, crea confianza y proporciona una fuente de diálogo favorable entre profesores y alumnos.

\section{Contexto del estudio}

Con la súbita interrupción de clases presenciales por COVID-19, las instituciones educativas iniciaron (y otras consolidaron) procesos de transformación en sus prácticas educativas mediante el uso de TIC y los ambientes digitales que estas proveen. Los elementos mencionados con anterioridad, son sin duda transferibles a cualquier contexto en el país, sin importar nivel educativo. La necesidad de praxis docente mediante la creación de diseños instruccionales que incluyan elementos adaptativos al inicio, durante y al final del proceso de enseñanza y que utilicen evaluaciones apropiadas acorde a los tiempos actuales es imperante. Lo anterior en beneficio de un aprendizaje significativo social y culturalmente. El desafío radica en generar estrategias pedagógicas significativas, desmarcadas de la educación bancaria, presencial o no, que Freire (1970:72) identifica como aquella en la cual "el único margen de acción que se ofrece a los educandos es el de recibir los depósitos, guardarlos y archivarlos".

Específicamente en el nivel de posgrado, las Instituciones de Educación Superior (IES) tienen claro que la pandemia revela desigualdades preexistentes, pero que, en este caso, al hablar de personas adultas económicamente activas en su mayoría, la adquisición y acceso a bienes culturales y materiales no es el tema de estudio en cuestión, no optamos por evaluar la vulnerabilidad en la que vive la comunidad estudiantil, sino de una cuestión inducida, construida socialmente, del capital humano interiorizado (Navarro et al., 2021) de aquellos aspectos del 
capital cultural que afectarán diferenciadamente a la facilitación no presencial de un curso, lo que puede explicar el incremento de la ansiedad y desmotivación de quienes participan en el proceso educativo; sus actitudes y desempeño al proceso, las estrategias, actividades y productos diseñados en el curso para el logro de los objetivos y la promoción de su aprendizaje.

Los procesos educativos en nivel posgrado establecen el desarrollo de competencias y adquisición de conocimientos, procedimientos y valores de forma autónoma, promoviendo el aprendizaje a través de actitudes crítico-reflexivas y que prioricen experiencias contextualizadas y colaborativas, con el objetivo principal de la creación de conocimiento para ser utilizadas en escenarios prácticos. Específicamente, el estudiante del Doctorado en Gestión Educativa y Políticas Públicas de la Universidad Autónoma de Zacatecas (DGEPP-UAZ), cuenta con un alto porcentaje de su tiempo en el trabajo, se dedica en su mayoría a la docencia, aunque también existen casos de puestos administrativos de poder (directores, supervisores, etc.). Así surge la necesidad de aprender asincrónicamente, utilizando como herramienta principal las TIC; sin embargo, las estrategias y prácticas apropiadas a los contextos educativos de posgrado, aún son tema de debate; éstas varían conforme a las tecnologías disponibles, los contenidos, los contextos y los conocimientos de los instructores, sin existir aún el referente definido institucionalmente para ser aplicado como modelo único.

Entre la diversidad de programas de posgrado que la Universidad Autónoma de Zacatecas (UAZ) ha creado para responder a la demanda de formación de recurso humano en áreas educativas se encuentra el Doctorado en Gestión Educativa y Políticas Públicas (DGEPP), que inició en enero de este año con clases a distancia y cuenta con un enfoque profesionalizante, es decir que conduce a la profundización y actualización de conocimientos, así como el desarrollo de competencias para el desempeño en un área o campo profesional.

La misión de los posgrados de la UAZ, plasmada dentro de las tareas de la Subcoordinación de Posgrado, es ofrecer una formación académica de alta calidad y generar cuadros técnicos, científicos y políticos competentes que cumplan con los estándares de calidad internacionales; además se plantea, que la educación superior a nivel de posgrado es la instancia formadora de recursos humanos y pieza fundamental para el desarrollo social. Así, el posgrado se debe caracterizar por ir a la vanguardia, ya que es el espacio donde se genera y difunde conocimiento a través de la investigación científica, misma que debe tener una aplicación social y ofrecer alternativas en la solución de problemas en distintos ámbitos; se espera que tales planteamientos sean retomados en cada uno de los programas de posgrado con sus vicisitudes que conforman el entramado de objetivos plateados por los programas académicos (Subcoordinación de Posgrado, 2019). 
De acuerdo con los lineamientos de la institución para el diseño de planes de estudios de posgrado, los fundamentos nacionales, regionales y locales, la factibilidad de la propuesta desde distintos rubros, así como el estudio de demanda estudiantil, se elabora este programa, dejando en claro los propósitos que persigue, el perfil del egresado, así como el diseño curricular necesario, cuyas características principales serán su flexibilidad curricular y sus áreas de énfasis terminal. Los objetivos, planteados por el núcleo académico de profesores (al cual pertenece la autora) de la formación del DGEPP son:

- Formar docentes e investigadores comprometidos, independientes, innovadores y creativos que conozcan, comprendan y expliquen los problemas de la educación desde las diversas perspectivas disciplinares y; herramientas metodológicas que sean capaces de proponer alternativas de solución.

- Formar investigadores que contribuyan a resolver las diversas problemáticas educativas, capaces de coordinar y formar parte de cuerpos y colectivos académicos que aborden la generación y aplicación del conocimiento, la administración del proceso educativo y los procesos institucionales de los programas, unidades y áreas académicas; así como investigadores capaces de proponer soluciones a los problemas educativos de las instituciones y los diversos niveles del sistema educativo de México, y la región. (Cervantes et al., 2019, p. 54).

El contexto específico del DGEPP actual es de una matricula total de 18 estudiantes (9 hombres y 9 mujeres) con edades que oscilan entre los 28 y los 40 años, cuentan con un trabajo en el ámbito educativo, en su formación de origen la mayoría es Licenciado en Educación o afines; fueron elegidos a partir de un proceso de selección que incluyó entrevista, presentación de documentos probatorios y $\mathrm{CV}$ y un curso propedéutico con una duración de tres meses. Todo ello lo hace un grupo con necesidades y expectativas acordes a un objetivo en común: estudiar un doctorado con orientación hacia la educación y las políticas públicas que la sustentan.

La asignatura Liderazgo y Gestión Educativa forma parte de los seminarios con carácter obligatorio a cursar en segundo semestre, está relacionada con las materias Problemas Contemporáneos de la Educación y Evaluación de la Calidad Educativa, su eje curricular es Tópicos Selectos de Políticas Públicas, cuenta con un total de 104hrs y 6 créditos. El propósito general es:

Conocer, identificar y comprender la conceptualización de gestión educativa tomando en cuenta sus dimensiones, enfoques y tendencias; analizar la importancia de un vínculo entre ésta y los fundamentos principales del liderazgo, entendiéndolo como el conjunto de procesos que orientan a las personas y a los equipos en una determinada responsabilidad institucional hacia el logro de la excelencia y el aprendizaje organizacional utilizando los principios de la administración, adoptando conductas incluyentes, desde la perspectiva de la sustentabilidad, y reconociendo la equidad como valor a fomentar en una educación para toda la vida. (De la Cruz, 2020, p. 2). 
Como puede apreciarse con las características mencionadas, las formas de organización y estructura que requiere la implementación de currículos con tendencia hacia la flexibilidad como este, suponen la incorporación de prácticas pedagógicas distintas, avanzar hacia un esquema donde exista mayor interacción del estudiante con los recursos diseñados por medio de tecnologías y, además, se priorice la expresión de ideas y argumentos en escenarios con sus pares. Ante estos retos, a continuación, se presenta el diseño instruccional que se implementó basado en los elementos teórico- contextuales desglosados.

\section{Estrategia metodológica}

En un marco de transformación de la docencia por las causas de la pandemia, la comprensión y aplicación de los principios de la Educación Adaptativa se vuelven imprescindibles, por tal motivo, la investigación basa sus resultados en la implementación de una Propuesta Curricular Adaptada para la asignatura de Liderazgo y Gestión Educativa que incluyera estrategias de enseñanza-aprendizaje, alineadas con evidencias (entregables como tareas o trabajos) que promovieran la reflexión del estudiante a través de sus diferentes estilos de aprendizaje. En particular se priorizó el pragmatismo al confrontar a los estudiantes con los conocimientos teóricos que marca el seminario y los conocimientos profesionales que poseen, profundizar en el actuar de su vida laboral y qué elementos pueden cambiar para mejorar sus contextos.

Así, se organizaron catorce sesiones teórico-prácticas, guiadas por dos docentes a través de videollamadas utilizando la aplicación Google Meet y sesiones de trabajo individual tutorizadas por distintos medios de comunicación síncrona y asíncrona (correos electrónicos, WhatsApp, chats, foros, etc.). El contenido del curso se diseñó en Moodle y puso a disposición del estudiante para su consulta de acuerdo a sus tiempos, la duración total del seminario es de quince semanas. En la siguiente tabla se resume la información:

Tabla 1. Organización del curso.

\begin{tabular}{|c|l|l|}
\hline No. Sesión & \multicolumn{1}{|c|}{ Objetivos } & \multicolumn{1}{c|}{ Contenidos } \\
\hline 1 & Bienvenida y presentación del seminario. & $\begin{array}{l}\text { Plan y programa, criterios de evaluaci- } \\
\text { ón, acuerdos. }\end{array}$ \\
\hline
\end{tabular}




\begin{tabular}{|c|c|c|}
\hline 2 & \multirow{4}{*}{$\begin{array}{l}\text { Identificar y comprender la conceptuali- } \\
\text { zación de gestión educativa, sus dimen- } \\
\text { siones, enfoques, procesos e instrumen- } \\
\text { tos para reflexionar sobre su función y } \\
\text { metas dentro de la comunidad y contexto } \\
\text { educativo, propiciando la comprensión } \\
\text { de situaciones problema y sus soluciones. }\end{array}$} & \multirow{4}{*}{ Unidad I. Gestión Educativa. } \\
\hline 3 & & \\
\hline 4 & & \\
\hline 5 & & \\
\hline 6 & \multirow{5}{*}{$\begin{array}{l}\text { Analizar y enunciar los fundamentos, } \\
\text { clasificación y teorías que determinan } \\
\text { el actuar de un buen líder, utilizando los } \\
\text { principios de la negociación y resolución } \\
\text { de conflictos para modificar y construir } \\
\text { ambientes de trabajo orientados hacia el } \\
\text { logro de los objetivos y el aprendizaje } \\
\text { eficaz. }\end{array}$} & \multirow{5}{*}{ Unidad II. Liderazgo. } \\
\hline 7 & & \\
\hline 8 & & \\
\hline 9 & & \\
\hline 10 & & \\
\hline 11 & \multirow{4}{*}{$\begin{array}{l}\text { Establecer la relación entre la gestión } \\
\text { educativa y liderazgo para proponer ins- } \\
\text { trumentos y procedimientos que le permi- } \\
\text { tan implementar propuestas en contextos } \\
\text { educativos orientadas hacia el logro de } \\
\text { una educación de calidad y enmarcadas } \\
\text { en los principios de la sana convivencia, } \\
\text { inclusión y justicia social. }\end{array}$} & \multirow{4}{*}{$\begin{array}{l}\text { Unidad III.- Liderazgo y gestión edu- } \\
\text { cativa para la inclusión y la justicia } \\
\text { social. }\end{array}$} \\
\hline 12 & & \\
\hline 13 & & \\
\hline 14 & & \\
\hline
\end{tabular}

Fuente: Elaboración propia.

El desarrollo del seminario "Liderazgo y Gestión Educativa" destacó de sus homólogos gracias a las siguientes características:

- Detección de necesidades de aprendizaje a través de la elaboración de una evaluación diagnóstica y adecuación de los objetivos y contenidos curriculares conforme a los resultados.

- Aprendizaje basado en problemas, evidencia final a través de un "Proyecto integrador" cuyo objetivo es la comprensión del vínculo entre gestión educativa y liderazgo para desarrollar propuestas que garanticen una educación de calidad, inclusión y justicia para todos, se elaboró durante el semestre integrando los conocimientos teórico-procedimentales conforme se avanzaba con las sesiones.

- Modelo invertido de aprendizaje, revisión de sustento teórico previo a las sesiones, priorizando en ellas procesos cognitivos de mayor complejidad, como la aplicabilidad de lo analizado con la realidad del estudiante y el trabajo colaborativo mediante el intercambio de opiniones con sus pares, para favorecer la argumentación crítica fundamentada desde un aprendizaje significativo.

- Estrategias de aprendizaje con entregables secuenciales (conocimientos anidados: necesidad del dominio de competencia 1 para la elaboración de evidencia 2 y así sucesivamente).

- Organización del escenario de aprendizaje simple, fácil de comprender y navegar en él, utilizando Moodle como herramienta facilitadora y estructurado a partir de tres secciones principales: (A) General. Avisos. (B) Descripción del curso. 
Bienvenida, objetivos y programa del curso, formas de evaluar y reglamento. (C) Unidades. Materiales y Actividades.

- Diseño de secuencia de actividades por unidad que incluía información detallada sobre las actividades a desarrollar, su objetivo, producto o evidencia, fecha de entrega y rúbrica con criterios e indicadores de evaluación.

- Sesiones adicionales a las programadas para tutorías individualizadas de acuerdo a tiempos de los estudiantes, lo anterior con el objetivo de proporcionar retroalimentación oportuna sobre los contenidos y actividades desarrolladas en el curso antes de la entrega del proyecto final y su calificación (evaluación formativa).

- Establecimiento de tiempos razonables de entrega y desarrollo de las actividades, siempre modificables a peticiones de los alumnos y acorde al nivel de dificultad.

- Implementación de estrategias (foros, exposiciones, documentos argumentativos) para el trabajo colaborativo donde se promueve la participación, el respeto a las ideas, la escucha asertiva y la evaluación entre pares (coevaluación).

- Elaboración de materiales didácticos adicionales para servir de apoyo durante las sesiones síncronas de aprendizaje. Así como información de recursos adicionales existentes en la web bajo diversos formatos (videos, podcast, artículos, libros, etc.) de apoyo para aquellos estudiantes que no dominen o tengan dudas sobre los conceptos.

- Sistema de evaluación continua que permitió prescindir de una prueba final, gracias a la distribución de la calificación definitiva entre los diferentes productos de aprendizaje, considerando entonces las actividades realizadas previamente para reducir el problema de garantía de autenticidad y posibilitar en todo momento la recuperación de la nota.

- Estrategias de enseñanza-aprendizaje que utilizaron las tecnologías ya disponibles en la Universidad y conocidas por los estudiantes (procesador de texto, editor de presentaciones, correo electrónico, Moodle) para que éstos se sintieran seguros al trabajar con elementos familiares y reducir la ansiedad de los entornos virtuales.

- Ambiente cordial en espacios de trabajo síncrono y asíncrono, priorizando la comunicación asertiva, el respeto a las ideas y la expresión de situaciones de crisis para promover e impulsar soluciones colectivas a desafíos urgentes que contribuyan a la transformación estructural de nuestro sistema educativo desde el actuar de los estudiantes.

En total se diseñaron 10 actividades de aprendizaje y el proyecto integrador, se utilizaron principalmente los recursos de Tareas y Foros para la interacción y entrega de las evidencias en Moodle. Los resultados se utilizarán para el análisis del desempeño de los estudiantes, así como insumos para los elementos a considerar en las conclusiones. 


\section{Análisis de resultados}

Una vez diseñadas e implementadas las estrategias de enseñanza-aprendizaje-evaluación descritas, es importante valorar sus resultados para tomar decisiones de modificación, mejora o abandono. El objetivo es novedoso y ambicioso porque implica realizar actividades divergergentes a las que nuestros estudiantes están acostumbrados, dejándolos a cargo de su proceso de aprendizaje, lo que implica cambios en sus actitudes y valores hacia los contenidos y seminario en general. Siendo coherentes con la teoría Adaptativa, las actividades y evidencias estuvieron diseñadas atendiendo a la diversidad, la evaluación se realizó al principio, durante y al finalizar la intervención educativa, desde una perspectiva para la formación y no como un medio de control o punitivo. En este apartado resumiremos los resultados más evidentes en torno a dos cuestiones: ¿El aprendizaje y desempeño de los estudiantes con la implementación de estrategias adaptativas en nivel posgrado? ¿Satisfacción de los alumnos por la metodología de enseñanza utilizada y los resultados obtenidos?

\section{La mejora de las competencias en los alumnos de posgrado relacionadas con las estrategias adaptativas}

Es importante sumar informaciones sobre los distintos productos, para mejorar el proceso y para comprobar la adecuación de los resultados a los intereses y necesidades de los alumnos y el contexto, lo anterior permite elevar la calidad del aprendizaje y aumentar el rendimiento de los alumnos tras la mejora de algunos elementos. Comencemos por analizar el desempeño en las actividades realizadas y los productos entregados por los estudiantes conseguidos gracias al informe general del espacio virtual del curso en Moodle: el 80\% de las actividades tuvieron un promedio de calificación igual o mayor a 9.1 de un total de 10 puntos posibles, el $90 \%$ de los estudiantes entregó sus productos en los tiempos establecidos, el promedio general del grupo fue de 8.92 tomando en cuenta los tiempos ordinarios del curso; sin embargo, este puntaje sube 9.31 una vez que se toman en cuenta las entregas tardías que, por motivos de salud y familiares, derivados de la contingencia sanitaria, dificultaron a un $10 \%$ de los estudiantes el cumplimiento conforme a lo acordado. El 100\% de los alumnos aprobaron el curso. Respecto al tiempo de permanencia de los estudiantes dentro de la plataforma para consultar materiales y subir evidencias de aprendizaje, éste tuvo un promedio de 4hrs semanales.

Otro aspecto que se indagó fue cuál actividad se destacó con el mejor puntaje, la información obtenida es muestra de que la motivación en el estudiante incrementa con la participación 
e interacción con sus compañeros, siendo la elaboración de una presentación y la exposición de manera grupal del tema "Los fundamentos del liderazgo (definición, importancia, clasificación y teorías), identificando las figuras y variables principales" la que obtuvo una calificación grupal promedio de 9.82 .

Cabe mencionar también que la actividad de aprendizaje con menor calificación fue el documento argumentativo que tenía como objetivo "Identificar las dimensiones y enfoques de la gestión educativa, analizando a detalle cuáles requieren de mayor atención dentro de su contexto y porqué" con una nota promedio grupal de 8.59 , los motivos principales proporcionados en la retroalimentación a los alumnos versaban en relación a fundamentación teórica endeble y necesidad de concretar las respuestas con ejemplos de su contexto, indicadores con valor específico informados en la rúbrica para dicha evidencia.

Finalmente, otro elemento relevante fue el desempeño en el proyecto integrador, éste incluyó información (antecedentes, misión, visión, valores, diagnóstico interno y externo, propuesta y enfoque pedagógico) de la institución educativa donde labora el estudiante, identificación y elaboración de un protocolo para la solución de una situación problémica identificada en su contexto. Es importante destacar la capacidad demostrada por los alumnos para responder rápida y eficientemente a los cambios generados por el entorno social en los diferentes niveles de concreción del sistema educativo, teniendo como base una argumentación fundamentada y un equilibrio entre la teoría y la práctica. La nota promedio grupal fue de 9.00.

\section{La resistencia pedagógica en posgrado}

La satisfacción es un buen indicador que muestra el grado de acuerdo del estudiante con las estrategias de enseñanza-aprendizaje-evaluación utilizadas durante el seminario. Para este aspecto, se ha recabado información proveniente de las sesiones de tutoría académica individuales, las opiniones expresadas en las videollamadas y las respuestas de retroalimentación de los estudiantes al concluir el seminario. La metodología implementada parece viable si se consideran los resultados de los estudiantes en cuestión a una evaluación sumativa; sin embargo, existieron manifestaciones de los mismos en el sentido de considerar su actuar dentro del curso demasiado, ya que para algunos era importante dar "mayor dirección e indicaciones" por parte del docente. Lo anterior pone en manifiesto un área de oportunidad con el estudiante adulto al reconocer que se requiere de un tiempo mayor para adaptarse a un rol más protagónico dentro de su aprendizaje. 


\section{Consideraciones finales}

El estado de confinamiento ocasionado por COVID-19 ha provocado transformaciones en los procesos educativos con adaptaciones apresuradas a pesar de que la educación adaptativa y la utilización de las TIC en este sector no es un tema nuevo; y aunque no existe una solución única y exenta de problemas y con garantía de cubrir todas las necesidades, la creatividad y capacidad de análisis y adaptación del profesorado es fundamental.

El diseño instruccional y método de evaluación de la asignatura Liderazgo y Gestión Educativa descritos se consideran innovadores ante el escenario que enfrentan la mayoría de los posgrados que insisten en mantener una base sólida de investigación no solo como soporte del programa sino como objetivo y exigencia de titulación, pero irrelevantes como preparación para el ejercicio profesional. Con los fundamentos teóricos y las características mencionadas es posible comenzar a sintonizar verdaderas acciones de respuesta, oportunas a las exigencias sociales y estar en condiciones de proponer soluciones aplicables desde el contexto y con las tecnologías disponibles. Su valor reside en que esta metodología permite tomar decisiones de planificación y valorar el proceso y los resultados obtenidos gracias a la combinación de componentes actitudinales y cognitivos, elementos que sugieren a otros profesores cómo podrían desarrollar ciertas estrategias que cuidadosamente planeadas y ejecutadas, son percibidas por los estudiantes como un medio que les ayuda a aprender.

De esta crisis debemos aprender y tomar las situaciones positivas en forma de lecciones aprendidas, el debate de los resultados sigue abierto, estamos en tiempos de flexibilidad, no de imposición, el establecimiento de acuerdos y redes colaborativas entre instituciones educativas a nivel global es ya una necesidad. Corresponderá a cada actor desde la gestión política, administrativa hasta la académica impulsar soluciones colectivas para reinventar el proceso educativo. ¿Por qué seguir haciendo lo mismo y esperar diferentes resultados?, dejemos de equipar las aulas con recursos tecnológicos y considerar que nuestros problemas se resolverán con mejor infratestructura para enseñar, mejor concentremos acciones para equipar a docentes y estudiantes con herramientas cognitivas para aprender, dinamicemos el proceso, comprendamos que los horarios, calendarios y lugares fijos se quedarán en la obsolescencia, ya que los individos enseñan y aprenden a todas horas. Estancarnos en las certezas de una normalidad sería un escenario utópico. Me gustaría concluir con una frase que leí alguna vez y de la cual desconozco el autor "La gente ya no busca líderes con maestría y doctorados; simplemente buscan líderes con corazón". 


\section{Referencias}

ÁLVAREZ-ÁLVAREZ, C. Teoría frente a práctica educativa: algunos problemas y propuestas de solución. Perfiles Educativos, 37 (148), p. 172-190, 2015. Disponível em: https://doi. org/10.1016/j.pe.2015.11.014. Acesso em: 20 nov. 2021.

ARETIO, L. G. Fundamentos y componentes de la Educación a Distancia. Revista Iberoamericana de Educación, 2(2), 43-61, 1999. Disponível em: http://revistas.uned.es/index.php/ ried/article/view/2076. Acesso em: 20 nov. 2021.

BELLOCH, C. Diseño instruccional. 2017. Disponível em: http://148.202.167.116:8080/jspui/bitstream/123456789/1321/1/EVA4.pdf. Acesso em: 20 nov. 2021.

BERAZA, M. A. Z. La atención escolar a la diversidad. Congreso Internacional Nuevas Orientaciones en la Atención a la Diversidad. Santiago de Compostela. Ponencia, 1999.

BOOTH, T. Index for inclusion. Developing learning and participation in schools. Bristol: CSIE, 2000. Disponível em: http://www.csie.org.uk/resources/inclusion-index-explained. shtml. Acesso em: 20 nov. 2021.

ARREDONDO, S. C.; DIAGO, J. C. Evaluación educativa de aprendizajes y competencias. Madrid, España: Pearson Educación, 2009. Disponível em: http://www.conductitlan.org. mx/07_psicologiaeducativa/Materiales/L_evaluacic3b3n_educativa_de_aprendizajes_y_competencias.pdf. Acesso em: 20 nov. 2021.

CEPAL. Informe sobre el impacto económico en América Latina y el Caribe de la enfermedad por coronavirus (COVID-19), 2020. Disponível em: https://repositorio.cepal.org/ handle/11362/45602. Acesso em: 20 nov. 2021.

CERVANTES, D.; ÁVILA, N.; ELÍAS, M.; DE LA CRUZ, Y.; CONEJO, R. Proyecto de Doctorado en Gestión Educativa y Políticas Públicas. Universidad Autónoma de Zacatecas, México, 2019.

DE LA CRUZ, Y. Liderazgo y Gestión Educativa: UDI. Universidad Autónoma de Zacatecas, México, 2020.

FREIRE, P. Pedagogía del oprimido. México: Siglo XXI Editores, 1970.

GARCÍA, M. G. Orientaciones para hacer viables las estrategias de adaptación en educación secundaria obligatoria. Revista de Orientación y Psicopedagogía, 11, 20, 229- 240, 2000. Disponível em: https://dialnet.unirioja.es/servlet/articulo?codigo=199833. Acesso em: 20 nov. 2021.

GARCÍA, M. G. MARTÍNEZ, B. A. La formación de competencias docentes para incorporar estrategias adaptativas en el aula. Revista complutense de educación, 19(2), 253-274, 2008. Disponível em: https://revistas.ucm.es/index.php/RCED/article/view/RCED0808220253A/15443. Acesso em: 20 nov. 2021.

GARCÍA-PEÑALVO, F. J. P.; CORELL, A.; ABELLA-GARCÍA, V.; GRANDE, M. La evaluación online en la educación superior en tiempos de la COVID-19. Education in the 
knowledge society: EKS, 2020. Disponível em: https://doi.org/10.14201/eks.23086. Acesso em: 20 nov. 2021.

GIRALDO, E. P. L. El diseño instruccional en la educación virtual: más allá de la presentación de contenidos. Educación y desarrollo social, 5(2), 112-127, 2011. Disponível em: https://dialnet.unirioja.es/servlet/articulo?codigo=5386237. Acesso em: 20 nov. 2021.

GONZÁLEZ-TEJERO, J. M. S., PARRA, R. M. P. La concepción constructivista de la instrucción. Hacía un replanteamiento del triángulo interactivo. Revista Mexicada de Investigación Educativa, 13 (38), 681-712, 2008. Disponível em: http:/www.scielo.org.mx/ scielo.php?script=sci_arttext\&pid=S1405-66662008000300002. Acesso em: 20 nov. 2021.

LATAS, A. P. Acerca del origen y sentido de la educación inclusiva. Revista de educación. Revista de Educación, núm. 327, 2002, p. 11-29. Disponível em: https://www.altascapacidades.es/webdocente/Educacion\%20inclusiva/lectura-15-Parrilla-Latas(2002).pdf. Acesso em: 20 nov. 2021.

MARTÍNEZ S. I. N.; MUÑOS, D. M. R.; FARRERA, R. F. M. Posgrado y educación no presencial. Argumentos. Estudios Críticos De La Sociedad, 2(96), 41-63, 2021. Disponível em: https://doi.org/10.24275/uamxoc-dcsh/argumentos/2021962-02. Acesso em: 20 nov. 2021.

ORTIZ, T. D. J. C.; RUBALCAVA, A. E. F.; CALDERÓN, D. V.; PEÑAFLOR, C. Z. Evaluando la enseñanza en el posgrado. Reencuentro. Análisis de problemas universitarios, (53), 63-84, 2009.

PARSONS, S. A.; VAUGHN, M. Toward adaptability: Where to from here? Theory Into Practice, 55, 267-274, 2016. Disponível em: https://doi.org/10.1080/00405841.2016.1173998 . Acesso em: 20 nov. 2021.

PARSONS, S. A.; VAUGHN, M.; SCALES, R. Q.; GALLAGHER, M. A.; PARSONS, A. W.; DAVIS, S. G.; PIERCZYNSKI, M.; ALLEN, M. Teachers' Instructional Adaptations: A Research Synthesis. Review of Educational Research, 88(2), 205-242, 2018. Disponível em: https://doi.org/10.3102/0034654317743198. Acesso em: 20 nov. 2021.

PRADO, R. A. La socioformación: un enfoque de cambio educativo. Revista Iberoamericana de Educación, 76(1), 57-82, 2018. Disponível em: https://doi.org/10.35362/rie7612955 Acesso em: 20 nov. 2021.

SHULMAN, L. S. Knowledge and Teaching: Foundations of the New Reform. Harvard Educational Review, 57 (1): 1-23, 1987.

SUBCOORDINACIÓN DE POSGRADO, U. Subcoordinación de Posgrado. Universidad Autónoma de Zacatecas, 2019. Disponível em: http://investigacion_posgrado.uaz.edu.mx/posgrado.Acesso em: 20 nov. 2021.

TOBÓN, S.; MARTÍNEZ, J. E.; VALDÉZ, E.; QUIRIZ, T. Prácticas pedagógicas: Análisis mediante la cartografía conceptual. Revista Espacios, 39(53), 2018. Disponível em: http:// www.revistaespacios.com/cited2017/cited2017-31.pdf. Acesso em: 20 nov. 2021. 
TYLER, R. W. Basic principle of curriculum and instruction. Chicago, USA: Chicago University, 1950.

ZABALZA, M.A. La atención escolar a la diversidad. Congreso Internacional Nuevas Orientaciones en la Atención a la Diversidad. Santiago de Compostela. Ponencia, 1999.

Recebido em: 30 de outubro de 2021.

Publicado em: 06 de dezembro de 2021. 